\title{
Chiral dynamos and magnetogenesis induced by torsionful Maxwell-Chern Simons electrodynamics
}

\author{
L. C. Garcia de Andrade ${ }^{\mathrm{a}}$ \\ Departamento de Física Teórica, IF-UERJ, Rua São Francisco Xavier 524, Maracanã, Rio de Janeiro, RJ CEP 20550, Brazil
}

Received: 5 January 2018 / Accepted: 7 March 2018 / Published online: 23 March 2018

(C) The Author(s) 2018

\begin{abstract}
Recently chiral anomalous currents have been investigated by Boyarsky et al. and Brandenburg et al. with respect to applications to the early universe. In this paper we show that these magnetic field anomalies, which can give rise to dynamo magnetic field amplification can also be linked to spacetime torsion through the use of a chemical potential and Maxwell electrodynamics with torsion firstly proposed by de Sabbata and Gasperini. When the axial torsion is constant this electrodynamics acquires the form of a Maxwell-Chern-Simmons (MCS) equations where the chiral current appears naturally and the zero component of torsion plays the role of a chemical potential, while the other components play the role of anisotropic conductivity. The chiral dynamo equation in torsionful spacetime is derived here from MSC electrodynamics. Here we have used a recently derived a torsion LV bound of $T^{0} \sim 10^{-26} \mathrm{GeV}$ and the constraint that this chiral magnetic field is a seed for galactic dynamo. This estimate is weaker than the one obtained from the chiral battery seed of $\sim 10^{30} \mathrm{G}$ without making use of Cartan torsion. The torsion obtained here was derived at $500 \mathrm{pc}$ coherence scale. When a chiral MF is forced to seed a galactic dynamo one obtains a yet weaker MF, of the order of $B \sim 10^{12} \mathrm{G}$, which is the value of a MF at nucleosynthesis. By the use of chiral dynamo equations from parity-violating torsion one obtains a seed field of $B \sim 10^{27} \mathrm{G}$, which is a much stronger MF closer to the one obtained by making use of chiral batteries. Chiral vortical currents in non-Riemannian spacetimes derived in Riemannian spaces previously by Flaschi and Fukushima are extended to include minimal coupling with torsion. The present universe yields $B \sim 10^{-24} \mathrm{G}$, still sufficient to seed galactic dynamos.
\end{abstract}

a e-mail: garciluiz@gmail.com

\section{Introduction}

Earlier Vilenkin [1] has shown that chiral massless fermions in parity-violating theories could generate chiral currents where the magnetic field would be proportional to that current under equilibrium conditions. That paper laid down the foundations of chiral dynamo theory as later developed by Froehlich and Pedrini [2] and by Boyarsky et al. [3], culminating in more recent investigations by Brandenburg et al. [4]. However, the main problem with the Vilenkin paper pointed out by himself is that when fermions are massive the flat chiral current vanishes. In this paper we show that really, as pointed out by Vilenkin, when one shifts to Kharzeev parityviolating QCD called local P-violation endowed with Cartan torsion, massive fermions do indeed give rise to a nonvanishing chiral current. Actually as is shown in the next section the chiral current axial torsion vector gives rise to an anisotropic tensor conductivity in chiral axial current when the chiral current can be shown to be expressed as the chiral component plus torsion contributions like the vector product between torsion vector and the magnetic field [5]. Previously Garretson et al. [6] have tried to obtain primordial magnetic fields (PMFs) that seed galactic dynamos without success by making use of an axion version of electrodynamics where the time derivative of the axion scalar fields plays the role of the chemical potential. In order to obtain a PMF of the order suitable to seed microgauss galactic magnetic fields (GMFs) via a dynamo mechanism we make use here of a MCCS version of electrodynamics and of a chiral dynamo equation derived from this electrodynamics to obtain a MF of the order of $B_{\mathrm{PMF}} \sim 10^{14} \mathrm{G}$. This has been obtained by making use of a LV torsion as obtained previously by the author [7] considering GMF at the scale of $500 \mathrm{pc}$. One of the main advantages of using torsion here is that we may generalize work and results by Boyarsky et al. in SM of particle physics to standard model extensions (SMEs) like LV [8,9] or supersymmetry, where torsion plays a major role. All these 
results are contained in Sect. 2. In Sect. 3 we address a brand new kind of chiral currents in $\mathrm{nR}$ spaces, which extends the recent result by Flasci and Fukushima [10] of chiral currents in Riemannian spaces, by minimal coupling with torsion, showing that the vortical $\mathrm{nR}$ expression appears in the second order - a microscopical result which does not appear in traditional Einstein Cartan gravity (EC) [11]. Other types of gravitational anomalies linked to gravity in torsionful space have been considered recently by Mavromatos and Plafitsis [12]. Section 3 addresses the problem of chirality and the determination of some primordial magnetic fields. Section 4 presents computations of chiral dynamos, taking into account the expansion of the universe in a Friedman universe. Discussions are left to Sect. 5, where we briefly present a very simple example of how to generalize gravitational anomalous currents in Riemannian spaces into non-Riemannian and show that a chiral magnetic effect can be obtained from a simple use of the Larmor frequency.

\section{Chiral dynamo equation from torsion modes in CS electrodynamics and magnetogenesis}

One simple manner to associate a chiral electric current to a spacetime torsion axial vector would be through the expression $J^{i} \mathrm{Ch} /$ torsion $\sim \epsilon^{i j k l} T_{j} F_{k l}$ where $i, j=1,2,3,4$ and $T_{j}$ is the torsion axial vector and $F_{i j}$ is the electromagnetic field tensor. This would be similar to the chiral current $J_{\mathrm{ch}}=\frac{\mu_{5} \alpha_{e m} B}{\pi}$, where $\mu_{5}$ is the net axial chiral chemical potential. Comparison of these last two equations yields a proportionality relation between the torsion and chemical chiral potential and half of our job could be ended. This case can also be particularly useful in the case investigated by Dvornikov and Semikoz [13] and its interaction with the case of electroweak interactions of chiral massless fermions, since they considered axions on that paper. Let us now show that this type of idea is present in some modified electrodynamics as the Garretson-Carroll-Field (GCF) axion scalar electrodynamics and the one we shall address here in detail which is a Chern-Simons electrodynamics with torsion given previously by de Sabbata and Gasperini based on the QED vacuum polarisation. The modified Faraday equation in GCF $\mathrm{ED}$ is

$\nabla \times \mathbf{E}+\nabla \times \mathbf{B}=4 \pi \mathbf{J}-\dot{\phi} \mathbf{B}+\nabla \phi \times E$

the last two terms on the RHS of this equation represent the influence of currents specially the first one of those would be the chiral like current $\mathbf{J} \sim \mu_{5} \mathbf{B}$ where $\mu_{5}$ is the chemical potential. Let us now review the MCCS equations of ED of de Sabbata and Gasperini and introduce the new concept of torsion like chirality in electrodynamics. In this sec- tion we shall be concerned with the axial anomaly in QED LV torsion modes, either with torsion trace or axial torsion, which is given by considering the de Sabbata and Gasperini $[14,15]$ photon polarization tensor QED. This axial anomaly in a certain sense can be called a quantum anomaly, by analogy. In general classical symmetries do not survive quantization. The most famous example is the SM axial anomaly and trace conformal anomaly, which in a Einstein-Cartan gravity framework has been investigated by Lucati and Prokopec [16]. First of all let us better understand why torsion can be associated to LV as shown by Kostelecky et al. [17]. This can be demonstrated by considering the Chern-Simons (CS) operator

$\mathcal{L}_{\mathrm{CS} / \mathrm{LV} / \mathrm{QED}}[A] \sim k_{\mu} A_{\nu} F^{\mu \nu}$

where $k_{\mu}$ is a constant vector associated with the LV parameter $b_{\mu}$, while the QED torsion LV interaction action can be expressed by

$\mathcal{L}_{\text {Torsion } / \mathrm{QED}}[A] \sim \epsilon^{\alpha \beta \mu \nu} T_{\mu} A_{\nu} F^{\alpha \beta}$

where $T_{\sigma}=\frac{1}{2} \epsilon_{\sigma \alpha \mu \nu} K^{\alpha \mu \nu}$ where is the axial torsion and where $K$ is the Cartan contortion. If one compares these last two expressions one notes that the vector $k_{\mathrm{LV}}$ plays a similar role of torsion $T$. Thus this analogy makes us conclude that Cartan torsion can be a faithful representation of the LV. LV could be interesting also in the early universe as can be seen in several recent papers. Now let us review in more detail the QED virtual pair of matter-antimatter, electron-positron pair decay, when photon is placed on a strong torsion field in the early universe for example. Matter-antimatter asymmetry is an important ingredient for the study of chiral anomalies where one handness type of fermions either massive or massless like neutrinos appears in different amounts in the universe. De Sabbata and Gasperini have long ago shown that if a photon, in second order perturbation of a QFT process, disintegrates into virtual pairs of matter-antimatter electronpositron like systems via the vacuum polarization effect, and since these particles are massive fermions, they couple with the Cartan torsion. They certainly justifies the presence of a torsional background. Thus the e.m. field itself is also affected by the presence of torsion. Actually even if torsion does not interact directly with the photon as advocated by Hehl et al. [18], it does interact quantum mechanically with a photon through a virtual pair of matter-antimatter. This is the reason most physicists believe that torsion in EinsteinCartan theory is a low energy version of a quantum torsion as investigated recently by Mavromatos and Plafitsis [12]. This theory would be a sort of quantum gravity with torsion, as investigated in detail by Shapiro [19,20]. This kind of process preserves gauge invariance and the second Maxwell equations modified by a quantum contribution of the order, having the classical field as a zeroth order which does not interact with torsion, and Maxwell equations acquire the clas- 
sical electrodynamical format. Since this lends torsion a QED quantum effect; it seems suitable to address the problems of quantum anomalies in spacetimes endowed with Cartan torsion modes. Now let us not go into details of de Sabbata Gasperini derivation of Maxwells equations using the QED approach. The invariant in the creation of virtual pairs generates an extra current

$J^{v}=\Pi^{v \mu} A_{\mu}$

which is anomalous since for a constant vacuum polarization tensor

$\partial_{\mu} J^{v}=\Pi^{v \mu} F_{\mu \nu}$

From an $\mathrm{S}$ matrix expansion with the electromagnetic propagator introduced by Feynman in his version of QED, which in vacuum is the polarization tensor, produces a charge renormalizable effect. In the perturbation one finds the polarization tensor in torsional spacetime as

$\Pi^{1}{ }_{\nu \mu}(p)=\frac{i e}{3 \pi^{2}} \epsilon_{\nu \mu \rho \sigma} T^{\rho} p^{\sigma}$

where $\mathrm{T}$ is again the axial torsion vector which couples naturally with fermion sector. Since $\Pi_{\mu \nu}^{1} p^{\nu}=0$ gauge invariance is preserved. Backing a coordinate transformation $i p \rightarrow \partial$ with the expression $F_{\mu \nu}=2 \partial_{[\mu} A_{\nu]}$ one obtains the Maxwell equations with torsion thus:

$\frac{1}{\sqrt{(-g)}} \partial_{\mu}\left(\sqrt{(-g)} F^{\nu \mu}\right)=4 \pi J^{\nu}+\frac{2 \alpha}{3 \pi} \epsilon^{\nu \mu \rho \sigma} F_{\mu \rho} T_{\sigma}$.

By making use of one set of Maxwell equations,

$\partial_{[\mu} F_{v \rho]}=0$,

one obtains the set of MCCS equations when torsion is constant. Note therefore that to restore the conservation of current or to cancel the anomaly the term $\partial_{[v} T_{\sigma]}$ must vanish, which implies that torsion is given by $T_{v}=\partial_{\nu} \phi_{\text {tors }}$ where $\phi_{\text {tors }}$ is the axion torsion potential so important in chiral dynamos. Now let us consider the expression above for the divergence of the current $\mathbf{J}$ and note that this expression is similar to the Chern-Simons Maxwell equation given by

$\partial_{\mu} F^{v \mu}=J^{v}-\frac{e^{2}}{\pi^{2}} P_{\mu} F_{\mu \nu}$

which shows that by comparing these two equations one reaches the conclusion that the axion vector $P_{\mu}=\partial_{\mu} \theta$ where $\theta$ is the axion scalar a prototype for dark matter particle, this induces one to realize that torsion vector is proportional to axion vector. Axions are very important for chiral dynamos. Investigating axions, quantum mechanics and mechanical pumping, Froelich and Pedrini [2] have shown that axions lead to instabilities in the magnetic field and it may be that when one considers galactic dynamos axions this may provide an explanation for large scale magnetic fields in the universe. Now to better investigate the magnetogenesis of this
MCCS electrodynamics let us write them down in $(3+1)$ dimensional form, which yields the Faraday equation

$\nabla \times \mathbf{E}=-\partial_{t} \mathbf{B}$,

the Coulomb modified equation

$\nabla . \mathbf{E}=4 \pi \rho+\frac{4 \alpha}{3 \pi}(\mathbf{T} . \mathbf{B})$,

and Ampère's equation

$\nabla \times \mathbf{B}=4 \pi \mathbf{J}+\frac{4 \alpha}{3 \pi}\left[T^{0} \mathbf{B}-\mathbf{T} \times \mathbf{E}\right]$.

Concerning the dependence of torsion this equation we show that on its right hand side one obtains the chiral like currents where the first of them simulates the chiral anomaly. It is important to note that Sivaram and De Sabbata put the 0component of the torsion to vanish, which actually probably led to them not considering this important analogy. Here we are considering that the magnetic monopole vanishes and $\operatorname{div} B=0$. Thus the chiral like dynamo equation from the above MCCS electrodynamics reads

$\partial_{t} \mathbf{B}=\nabla \times\left(\mathbf{v}(\times \mathbf{B})-\eta T^{0} \nabla \times \mathbf{B}\right.$

and by substitution of the Bianchi identity,

$\nabla \times \mathbf{B}=k \mathbf{B}$,

into the last form of dynamo equation one obtains the interesting form of the dynamo equation

$\partial_{t} \mathbf{B}=\nabla \times\left(\mathbf{v}(\times \mathbf{B})-\eta T^{0} k \mathbf{B} ;\right.$

to arrive at Eq. (13) we make use of the Lorentz relation

$\mathbf{E}=\mathbf{v} \times \mathbf{B}+T^{0} \eta \mathbf{B}$.

Here $\alpha$ is the fine structure constant. Let us now solve the chiral dynamo equation obtained from MCCS electrodynamics dimensionally. As we are only interested in the torsion effects on chiral magnetogenesis, the chiral dynamo equation reduces to

$\partial_{t} B \sim \eta T^{0} k B$

where $B$ is the modulus of the MF. Since the wave vector $k$ is given by the inverse of the coherent length $L$, one may reexpress this formula as

$\partial_{t} B \sim \eta \frac{T^{0} B}{L}$.

The solution of this equation approximately reads

$B \sim B_{\text {seed }} \eta \frac{T^{0}}{L} t$.

By the above galactic magnetism data one obtains for a seed field of the chiral battery $B \sim 10^{14} \mathrm{G}$. Now by considering that the B field is the GMF of microgauss order one obtains from the same chiral dynamo equation that $B \sim 10^{12} \mathrm{G}$ as 
of today. This galactic dynamo seed is of the order of the nucleosynthesis MF. In this section we have not taken into account the expansion of the universe, nor the value of the chemical potential in simulations. In the next section these data shall be taken into account.

\section{Small-scale dynamo equation from axial anomalies with torsion}

Parity violation in gravity has been investigated recently by Mukhopadhayaya and Sen [21], who concluded that considering torsion-axion duality arising in a string scenario via Kalb-Ramond field [22] leads to parity-violating interactions for spin- $\frac{1}{2}$ fermions. This gives us one of the main motivations to investigate the breaking of symmetries or chiral anomalies in spacetimes endowed with torsion; actually not only the axial torsion but also trace torsion vector couples with the fermion which in later sections of this paper shall allow one to obtain the dynamical equations for the charge asymmetry in the early universe between left- and right-chiral fermions. The gravitational anomalies are not only related to $\mathrm{P}$ parity violation as addressed by Boyarsky et al. [10] but also to the breaking of Lorentz symmetry investigated by Kostelecky et al. [11] and more recently by the author [12] in the context of chiral dynamos as investigated by Brandenburg et al. [4] in the context of general relativistic cosmology. In this section we shall derive the small-scale dynamo equation. In the case of a Minkowski relativistic spacetime Joyce and Shapochnikowski [23] showed that $j^{\mu}{ }_{R}$ and $j^{\mu}{ }_{L}$ ( $\mu=0,1,2,3)$ together are non-conserved asymmetric due to the Adler anomaly; we have the expression

$\partial_{\mu}\left(j_{R}^{\mu}-j_{L}^{\mu}\right)=\frac{e^{2}}{2 \pi^{2}}(\mathbf{E} . \mathbf{B})$.

Note that unless the electric and magnetic fields are orthogonal, the Adler anomaly is obeyed. Let us now consider the minimal coupling between Minkowski spacetime and torsion as $\partial_{\mu} \rightarrow \partial_{\mu}+T_{\mu}$ which upon substitution in the general form of the Adler anomaly [24],

$\partial_{\mu}\left(j_{R}^{\mu}-j_{L}^{\mu}\right)=\frac{e^{2}}{2 \pi^{2}}\left(F_{\mu \nu} \cdot \tilde{F}^{\mu \nu}\right)$,

yields

$\partial_{\mu}\left(j^{\mu}{ }_{5}\right)=\frac{e^{2}}{2 \pi^{2}}\left(F_{\mu \nu} \cdot \tilde{F}^{\mu \nu}+i T_{\mu}\left(j_{R}^{\mu}-j_{L}^{\mu}\right)\right)$.

Here $j^{\mu_{5}}=\left(j^{\mu}{ }_{R}-j^{\mu}{ }_{L}\right)$. Actually one could understand $\nabla_{\mu \text { torsion }} j_{\mu_{5}}=0$ which is easily obtained when a classical anomaly is considered in contrast to quantum anomalies. Here $\nabla_{\text {torsion }}$ is the $3 \mathrm{D}$ covariant derivative or grad operator. Therefore the classical anomaly can be canceled in contorted spacetime if one considers that this anomaly cancelation,

$\partial_{\mu}\left(j_{R}^{\mu}-j_{L}^{\mu}\right)=0$,

leads to the following constraints between electric and magnetic fields and axial torsion:

$F_{\mu \nu} \cdot \tilde{F^{\mu \nu}}=-i \frac{e^{2}}{2 \pi^{2}} T_{\mu}\left(j_{R}^{\mu}-j_{L}^{\mu}\right)$.

Now let us derive the small-scale chiral dynamo equation in contorted spacetime based on the equations by De Sabbata and Sivaram,

$$
\begin{aligned}
\nabla . \mathbf{E} & =4 \pi e n+\frac{4 \alpha}{3 \pi}(\mathbf{T} . \mathbf{B}), \\
\nabla \times \mathbf{B} & =\frac{4 \alpha}{3 \pi}(\mathbf{E} \times \mathbf{T})+4 \pi \mathbf{j},
\end{aligned}
$$

which is the Ampère equation, and the monopole free equation

$\nabla \cdot \mathbf{B}=0$

and the Faraday equation in Cartan space

$\nabla \times \mathbf{E}=-\partial_{t} \mathbf{B}$

These were derived from fermion-anti-fermions interactions. By making use of the constitutive equations of the CME electric current jCME [4],

$e j_{\mathrm{CME}}=\frac{2 \alpha \mu_{5}}{2 \pi^{2}} \mathbf{B}+\sigma \mathbf{E}$

where $\sigma$ is the electrical conductivity and $\mu_{5}$ is the chiral chemical potential treated here as constant in the approximation where the universe is at a temperature $T \leq T_{R} ; T_{R}$ is the right chirality electron temperature as shown by Joyce and Shaposhnikov [23], or more recently by Rogachevskii et al. [25] using chiral MHD. Taking the curl of the last expression one obtains

$e \nabla \times j_{\mathrm{CME}}=\frac{2 \alpha \mu_{5}}{2 \pi^{2}} \nabla \times \mathbf{B}+\sigma \nabla \mathbf{E}$.

Thus by substituting the above Faraday equation into this expression one obtains the chiral dynamo equation in contorted spacetime

$\nabla \times(\mathbf{v} \times \mathbf{B})-4 \pi e j_{\mathrm{CME}}=\partial_{t} \mathbf{B}$

Though this is a very interesting equation on its own, it does not contain explicitly the torsion axial vector. To introduce it we consider the expression

$\nabla \times(\mathbf{v} \times \mathbf{B})+4 \frac{\alpha}{3 \pi} \mathbf{E} \times \mathbf{T}-\nabla \times \mathbf{B}=\partial_{t} \mathbf{B}$

We have the Ohm law,

$\mathbf{E}=\frac{e}{\sigma} j_{\mathrm{CME}}-\frac{\alpha}{2 \pi^{2} \sigma} \mu_{5} \mathbf{B}$. 
Substitution of this into (32) yields

$\nabla \times(\mathbf{v} \times \mathbf{B})+4 \frac{\alpha e}{3 \pi \sigma} \mathbf{j}_{\mathrm{CME}} \times \mathbf{T}+\frac{2 \alpha^{2}}{3 \pi^{3}} \sigma \mu_{5} \mathbf{T} \times \mathbf{B}=\partial_{t} \mathbf{B}$

To find a simpler form for the chiral dynamo equation one assumes that

$\mathbf{j}_{\mathrm{CME}} \times \mathbf{T}=0$,

which upon substitution into Eq. (34) yields

$\nabla \times(\mathbf{v} \times \mathbf{B})+\frac{2 \alpha^{2}}{3 \pi^{3} \sigma} \mu_{5} \mathbf{T} \times \mathbf{B}=\partial_{t} \mathbf{B}$,

which is the final form for the chiral dynamo equation in terms of axial torsion. A Fourier spectrum of this equation yields

$i \mathbf{k} \times(\mathbf{v} \times \mathbf{B})+\frac{2 \alpha^{2}}{3 \pi^{3} \sigma} \mu_{5} \mathbf{T} \times \mathbf{B}=\partial_{t} \mathbf{B}$,

which yields the solution for the chiral dynamo amplification of the magnetic field,

$\partial_{t} \mathbf{B}=\left(i k v+\frac{2 \alpha^{2}}{3 \pi^{3} \sigma} \mu_{5} T\right) \mathbf{B}$,

which upon integration yields

$B=B_{\text {seed }} \exp \left(\left[i k v+\frac{2 \alpha^{2}}{3 \pi^{3} \sigma} \mu_{5} T\right] t\right)$.

Throughout this work we use the cosmic time. Note that for the present universe where conductivity is large and torsion is weak, of the order of $T \sim 10^{-26} \mathrm{GeV}$, the second term inside the brackets can be dropped in comparison to the oscillating term, and the magnetic field oscillates; however, in the case of early universe where the torsion mass, for example, is of the order of $200 \mathrm{GeV}$ one obtains a growing mode for the magnetic field which represents the torsion contribution to small-scale chiral dynamos. The final expression becomes

$B=B_{\text {seed }}\left(\left[\frac{2 \alpha^{2}}{3 \pi^{3} \sigma} \mu_{5} T t\right]\right)$.

Let us now use simulations due to Schoeber et al., which are the chemical potential $\mu_{5} \sim 10^{-4}$ the conductivity $\sigma \sim 100$ and the above estimate for the torsion, and one obtains $B \sim 10^{27} \mathrm{G}$, which is much stronger than the seed field obtained in the last section. We here also use the chiral battery field as a seed field. This shows that the chiral dynamo yields stronger MF closer to the ones obtained by Ahonen and Enqvist. Note by computing B nowadays for the present universe yields $B \sim 10^{-24} \mathrm{G}$, which is still sufficient to seed galactic dynamos. In the next sections we address torsion interaction with chiral fermions in the Friedman universe, which however, we do not consider as expanding to reinforce and stress the role of torsion into the chiral dynamo in this universe.

\section{Axial anomalies with torsion trace vectors in Friedmann universe}

In this section we shall consider the solution the equations of axial anomalies in Friedman universe and chiral dynamos and its efficiency on a torsion background. Let us start by magnetic fields in the Friedmann universe in general relativistic cosmology dynamos

$\dot{\mathbf{B}}=-2 H \mathbf{B}$,

where $H$ is the Hubble parameter, a is the expansion of the universe. The metric is given by

$\mathrm{d} s^{2}=\left(\mathrm{d} t^{2}-a^{2} \mathrm{~d} \mathbf{x}^{2}\right)$

or in the conformal time $\tau$ form

$\mathrm{d} s^{2}=a^{2}(\tau)\left(\mathrm{d} t^{2}-\mathrm{d} \mathbf{x}^{2}\right)$,

where for the relation between the two times one has $\mathrm{d} \tau=$ $a^{-1} \mathrm{~d} t$. Now let us consider in this section the Maxwell chiral electrodynamics in a spacetime with metric determinant $\mathrm{g}$, which is given by

$\frac{1}{\sqrt{-g}} \partial_{\mu}\left(\sqrt{-g} j^{\mu}\right)=\frac{\alpha_{e m} \epsilon^{\mu \nu \rho \lambda}}{4 \pi \bar{h}} F_{\mu \nu} F_{\rho \lambda}$.

Now let us substitute this Maxwell chiral equation by making use of the minimal coupling, which will give rise naturally to the torsion trace, as

$D_{\mu} j^{\mu}=\partial_{\mu} j^{\mu}+\Gamma_{\mu} j^{\mu}+K_{\mu \rho}^{\mu} j^{\rho}$

where here $\Gamma_{\mu}$ is the Riemann-Christoffel symbol of general relativity contracted and $K_{\mu}=T_{\mu}$ is the spacetime contortion tensor contracted, which coincides with the torsion trace $T_{\mu}$. Note that the axial torsion vanishes in this case since

$K_{\mu \rho}^{\mu}=\epsilon_{\mu \rho \sigma}^{\mu} K^{\sigma}=0$,

which vanishes identically since the $\epsilon$ tensor is totally skewsymmetric. Thus the chiral Maxwell equation in curved spacetime with torsion can be written as

$\frac{1}{\sqrt{-g}} \partial_{\mu}\left(\sqrt{-g} j_{\mathrm{CME}^{\mu}}\right)=\sqrt{-g}\left[\frac{\alpha_{e m} \epsilon^{\mu \nu \rho \lambda}}{4 \pi \bar{h}} F_{\mu \nu} F_{\rho \lambda}-T_{\mu} j^{\mu}\right]$.

Therefore we now can obtain an anomaly cancelation by considering that the term inside the bracket in the last expression vanishes; this leads to a severe constraint in the torsion trace and actually for an expression for the chiral current $j_{\mathrm{CME}}$ in terms of torsion trace vector. These expressions are

$\frac{1}{\sqrt{-g}} \partial_{\mu}\left(\sqrt{-g} j_{\mathrm{CME}}{ }^{\mu}\right)=0$

and

$\frac{\alpha_{e m} \epsilon^{\mu \nu \rho \lambda}}{4 \pi \bar{h}} F_{\mu \nu} F_{\rho \lambda}=T_{\mu} j^{\mu}$. 
From this last expression one obtains the constraints between the torsion trace vector and the chiral currents,

$T_{\rho} j^{\rho}=\frac{\alpha_{e m}(\mathbf{E} . \mathbf{B})}{4 \pi h}$.

Similar expressions can be obtained from the nonconservation of the chiral charge number $n_{5}$ if one considers the following transformations on the electric and magnetic fields from the Hubble expansion $a(\tau)$ where $\tau$ is the conformal time parameter given by

$\mathrm{d} \tau=\frac{\mathrm{d} t}{a(t)}$,

and the conformal metric is given by

$\mathrm{d} s^{2}=a^{2}\left(-\mathrm{d} \tau^{2}+\mathrm{d} \mathbf{x}^{2}\right)$.

Now let us write down the form of the e.m. field tensor $\mathrm{F}$ in terms of the Hubble parameter $a(\tau)$. Thus

$F_{i j}=a^{2} \epsilon_{i j k} B^{k}$,

$F_{0 j}=a E^{j}$,

where $(i, k=1,2,3)$. The Maxwell equation in an expanding universe reads

$\nabla_{\mu} F^{\mu \nu}=\frac{4 \pi}{c} j^{v}$

where $\nabla$ is the covariant derivative operator in Riemannian spaces. Therefore the expression for the 0-component of the Maxwell equation is

$a^{3}\left(\frac{\partial j_{0}}{\partial t}+3 H \rho+\nabla \cdot \mathbf{j}\right)=0$.

Now let us to present the equation for $j^{5}$, which is

$\frac{\partial\left(n_{5} a^{3}\right)}{\partial t}+\nabla \cdot\left(a^{3} j^{5}\right)-a T j^{5}=\frac{2 \alpha_{e m} a^{3}(\mathbf{E} . \mathbf{B})}{\bar{h}}$.

Transforming this equation to conformal coordinates yields

$\frac{\tilde{\partial} \tilde{n_{5}}}{\partial t}+\tilde{\nabla} \cdot\left(\tilde{j^{5}}\right)-\tilde{T} \tilde{j}^{5}=\frac{2 \alpha_{e m} a^{3}(\tilde{\mathbf{E}} \cdot \tilde{\mathbf{B}})}{\bar{h}}$.

Simulations of these equations may appear elsewhere.

\section{Discussions and conclusions}

Torsion fields introduced in CP-violating cosmic axion $\alpha^{2}$ dynamos by the author [26] in order to obtain Lorentz violating bounds for torsion were revisited. Oscillating axion solutions of the dynamo equation with torsion modes were obtained taking into account dissipative torsion fields. Magnetic helicity torsion oscillatory contributions were also obtained. In this paper we use chiral global anomaly ideas of Zanelli and Chandia expressed as the topological invariant $<j^{5}>\sim \int F \wedge F$ where $F=d A$, and we transport them to cosmological axial anomalies in a contorted Friedmann universe, while this universe is isotropic and we deal with the early universe, another work in progress would be to consider the Maxwell chiral equations in a anisotropic spacetime as Bianchi type I solutions for example. Other interesting nice properties of gravitational anomalies are the $\int R^{\mu}{ }_{v} \wedge R^{v}{ }_{v}$ applied to computed gravitational anomalies associated to these spaces and one might consider the chiral dynamos [4] associated to them. The investigations here may pave the way to building models for quantum gravity based on torsion as well as quantum anomalies. In the remaining of the discussion we shall briefly show how to transform Flaschi-Fujikawa gravitational and chiral anomalies in Riemannian space to non-Riemannian. Take for example the simplest example of the CS current vortical current $j_{A} \sim-\omega R$ where $\omega$ is the frame rotation and $R$ is the Ricci tensor. The new current formula in terms of the torsion trace becomes $j_{A} \sim-\omega\left(R-T^{2}\right)$. Note that if one takes into account the Larmor frequency formula and substitutes in this expression the important chiral magnetic effect, that would appear in this anomalous current as $j_{A} \sim T^{2} B$ where $B$ is the magnetic field. This is of course very similar to the formulas we discuss above in MCCS electrodynamics. The basic difference is that now that the dependence on the torsion is quadratic and not linear. It is interesting how a result of nonRiemannian gravitational anomaly gave rise to a CME. More investigations are actually the subject of a recent preprint, called non-Riemannian gravitational and mixed anomalies [27-33], and we study their implications to astrophysics and cosmology. It is still in preparation. Emphasis shall be given to topological induced currents. In most of the computations in magnetogenesis we use data from the estimates of chiral batteries [34]; see also work by Ahonen and Enqvist [35]. Just before completion of this paper we were told about the paper of Cesare et al. [36] where leptogenesis induced by torsion is analyzed and a very weak torsion is called to attention being the cause of non-detectability at the $\mathrm{CMB}$ signatures. Torsion bounds are consistent with the bounds used here to investigate magnetogenesis induced by torsionful electrodynamics. Their torsion upper bound is of the order of $T \sim \mathcal{O}\left(10^{-11}\right) \mathrm{GeV}$, which is consistent with the bounds used here. Ending our discussions, one could say that our estimates in chiral magnetogenesis are consistent with previous computations, which give support and strength to torsionful theories, not only the ones of the string type by inspiration of the Kalb-Ramond torsion, but also at the lower energy limit of Einstein-Cartan gravity and of torsionful Maxwell equations. In the QCD chiral case it is easy to show that by minimally coupling torsion with the non-Abelian gauge field one obtains a massive gauge particle where the square of the torsion induces mass [37]. The consequences of these torsion massive Yang-Mills bosons to cosmology from chiral anomalies may be further investigated. Recently Schober 
et al. [38], by using numerical simulations, have obtained $B_{C h} \sim 10^{-18} \mathrm{G}$ at $1 \mathrm{Mpc}$ scales. This result is stronger than the result obtained here at the same scale but it has the advantage of being analytical and coming with the strong simplification of a constant chiral chemical potential. Note that previously one [39-44] used several mechanisms to obtain results in magnetogenesis in torsionful spacetimes. Unfortunately in most of these papers one did not address the chiral magnetogenesis, problems with the breaking of gauge invariance and the introduction of massive photons to say the least. In one of the papers with torsion, no dynamo mechanism is achieved to seed galactic dynamos.

Acknowledgements We would like to express gratitude to A. Boyarsky and J. Schober for helpful discussions on the subject of this paper. We also thank colleagues of the workshop on Magnetic Fields in the Universe at the international institute of physics. Special thanks go to E. Mielke and F.W. Hehl for long conversations on EC gravity and chiral anomalies over the past three decades. Financial support from the University of State of Rio de Janeiro (UERJ) is gratefully acknowledged.

Open Access This article is distributed under the terms of the Creative Commons Attribution 4.0 International License (http://creativecomm ons.org/licenses/by/4.0/), which permits unrestricted use, distribution, and reproduction in any medium, provided you give appropriate credit to the original author(s) and the source, provide a link to the Creative Commons license, and indicate if changes were made. Funded by SCOAP ${ }^{3}$.

\section{References}

1. A. Vilenkin, Phys. Rev. D 21, 2260 (1980)

2. J. Froehlich, B. Pedrini, Axions. Quantum mechanics and pumping. Cond. Mat. 021236 (2002)

3. A. Boyarsky, O. Ruchaysky, M, Shaposhnikov, Long range magnetic fields in the ground state of the SM plasma model. arXiv:1204.3604 [hep-ph]

4. A. Brandenburg, J. Schober, I. Rogacheveskii, T. Kahniashvili, A. Boyarsky, J. Froelich, O. Ruchaykiy, N. Kleeorin, The turbulant chiral-magnetic cascade in the early universe. Astrophys. J. Lett. 845, L21 (2017)

5. L.C. Garcia de Andrade, Gravitational anomalies and chiral dynamos in non-Riemannian spaces, EUR libre editions (2018) (in press)

6. W. Garretson, G.B. Field, S.M. Carroll, Phys. Rev. D 46, 5346 (1992)

7. L. Garcia de Andrade, Eur. Phys. J. C 77, 401 (2017)

8. A. Kosteleckly, Phys. Rev. D 69, 105009 (2004)

9. A. Kostelecky, M. Mewes, APJ 689, L1 (2008)

10. A. Flachi, K. Fukushima, Chiral vortical effect in curved space and the Chern-Simons current. arXiv:1702.04753v1 [hep-ph]

11. V. De Sabbata, C. Sivaram, Spin and torsion in gravitation (World Scientific, Singapore, 1997), pp. 44-57

12. N. Mavromatos, A. Pilaftsis, Anomalous majorana neutrino masses from torsionful quantum gravity (2012). arXiv:1209.6387v2 [hep$\mathrm{ph}]$
13. M. Dvornikov, V. Semikoz, Chiral magnetic effects in the presence of electroweak quasiclassical phenomena (1997). arXiv: 1702.06426

14. V. de Sabbata, M. Gasperini, Phys. Rev. D 23, 2116 (1981)

15. M. Joyce, M. Shaposhinikov, Primordial magnetic fields, right electrons and abelian anomaly. Phys. Rev. Lett. 79, 1193-1196 (1997)

16. S. Lucati, P. Prokopec, Conformal trace anomaly in CartanEinstein gravity. Los Alamos arxives preprints (2017)

17. A. Kostelecky, N. Russell, J. Tasson, Phys. Rev. Lett. 100, 111102 (2008)

18. F.W. Hehl, J.D. McCrea, E. Mielke, Y. Neémann, Phys. Rep. 258, 36 (1995)

19. I.L. Shapiro, Physical aspects of spacetime torsion. Phys. Rep. 57, $2(2002)$

20. I. Rogachevskii, T. Kahniashvili, A. Boyarsky, J. Froelich, O. Ruchaysky , N. Kleeorin, A. Brandenburg, J. Schober, Laminar and turbulent dynamos in chiral magnetohydrodynamics I. Theory (2017). arXiv:1705.00378v1

21. B. Mukhopadhayaya, S. Sur, Mod. Phys. Lett. A 2002, 47 (2009)

22. M. Kalb, P. Ramond, Phys. Rev. D 9, 2273 (1974)

23. M. Joyce, M. Shaposhinikov, Primordial magnetic fields, right electrons and abelian anomaly. CERN/Th/97-31 and astro-ph/9703005

24. S. Adler, Phys. Rev. 177, 2426 (1997)

25. I. Rogachevsky, O. Ruchayskiy, A. Boyarskii, J. Froelich, N. Kleeorin, A. Brandenburg, J. Schober, Laminar and turbulent dynamos in chiral MHD-I: theory. Los Alamos arXives (2015)

26. L. Garcia de Andrade, Mod. Phys. Lett. A 26, 11 (2011)

27. K. Landsteiner, Phys. Rev. B 89, 075124 (2014)

28. J. Zanelli, O. Chandia, Torsional topological invariants. arXiv:hep-th/9708138v2

29. Y. Obukhov, E. Mielke, J. Budczies, F.W. Hehl, On the Chiral anomaly in non-Riemannian spacetimes. e-print gr-qc/9702011

30. H.T. Nieh, M. Yan, Ann. Phys. 138, 237 (1997)

31. E. Mielke, E.S. Romero, Phys. Rev. D 73, 043521 (2008)

32. E.W . Mielke, Anomalies and gravity (2006). arXiv:0605.159v1 [hep-th]

33. A. Dobado et al., Effective Lagrangeans in the SM (Springer, Berlin, New York, 1992)

34. S. Anand, J. Bhatt, A. Pandey, Chiral, scaling laws and magnetic fields (2017). arXiv:1705.0368v2 [astroph-Co]

35. J. Ahonen, K. Enqvist, Magnetic field generation in first orderphase transition bubbles (1997). arXiv:hep-ph/9704334v1

36. M. de Cesare, N. Mavromatos, S. Sarkar, Eur. Phys. J. C 75(10), $514(2015)$

37. L.C. Garcia de Andrade, Yang-Mills gravity, chiral fermions and torsional anomalies. Submitted to Class and Quantum Gravity (2017)

38. J. Schober, I. Rogachevsky, O. Ruchayskiy, A. Boyarskii, J. Froelich, N. Kleeorin, A. Brandenburg, Laminar and turbulent dynamos in chiral MHD-II: simulations. Los Alamos. arXiv:1711.09733v1 [phys-flu-dyn]

39. L.C. Garcia de Andrade, Class. Quantum Gravity 34, 205010 (2017)

40. L.C. Garcia de Andrade, Phys. Lett. B 711, 143 (2012)

41. L.C. Garcia de Andrade, Phys. Lett. B 468, 28 (2011)

42. L.C. Garcia de Andrade, Broken symmetries in space-time with torsion and galactic magnetic fields without dynamo amplification. Int. J. Astron. Astrophys. (2012) (in press)

43. L.C. Garcia de Andrade, Mod. Phys. Lett. A 26, 11 (2011)

44. V. de Sabbata, L.C. Garcia de Andrade, C. Sivaram, Int. J. Theor. Phys. 32(9), 1523 (1993) 